\title{
Two-dimensional electron gas oxide remote doping of $\mathrm{Si}(001)$
}

\author{
Eric N. Jin, ${ }^{1}$ Arvin Kakekhani, ${ }^{2}$ Sohrab Ismail-Beigi, ${ }^{1,3}$ Charles H. Ahn, ${ }^{1,3}$ and Frederick J. Walker ${ }^{1}$ \\ ${ }^{1}$ Department of Applied Physics, Yale University, New Haven, Connecticut 06511, USA \\ ${ }^{2}$ Department of Chemical Engineering, Stanford University, Stanford, California 94305, USA \\ ${ }^{3}$ Department of Physics, Yale University, New Haven, Connecticut 06511, USA
}

(Received 17 May 2018; published 26 November 2018)

\begin{abstract}
We demonstrate the integration of a two-dimensional electron gas (2DEG) oxide structure composed of $\mathrm{LaTiO}_{3} / \mathrm{SrTiO}_{3}$ (LTO/STO) on undoped $\mathrm{Si}(001)$ that possesses the attractive attributes of high charge density from the oxide and high mobility of silicon. Key to this approach is modification of the oxygen content at the STO-Si interface, which tunes the band alignment and induces electron carriers to move from the oxide 2DEG to form a 2DEG in the silicon substrate. As a consequence, the overall mobility of the heterostructure increases by two orders of magnitude compared to that in the oxide $2 \mathrm{DEG}$ to up to $100 \mathrm{~cm}^{2} \mathrm{~V}^{-1} \mathrm{~s}^{-1}$ at room temperature, with a carrier density of $\sim 1 \times 10^{13} \mathrm{~cm}^{-2}$. This approach can be applied to technologies that require both high carrier and high carrier mobility for applications in plasmonics and high power electronics.
\end{abstract}

DOI: 10.1103/PhysRevMaterials.2.115001

\section{INTRODUCTION}

The discovery of oxide two-dimensional electron gas (2DEG) systems such as $\mathrm{LaAlO}_{3} / \mathrm{SrTiO}_{3}$ (LAO/STO) [1] and $R \mathrm{TiO}_{3} / \mathrm{SrTiO}_{3}(R \mathrm{TO} / \mathrm{STO})$ [2-6], where $R$ is a trivalent rare earth ion, has opened a new field of physics for high density charge modulation [7] and the development of devices built from oxide heterostructures. While STO-based structures have demonstrated high electron mobilities at low temperature $[8,9]$, their mobilities are limited by phonon scattering to $6 \mathrm{~cm}^{2} \mathrm{~V}^{-1} \mathrm{~s}^{-1}$ at $300 \mathrm{~K}[10]$ and are lower than conventional semiconductors such as $\mathrm{Si}$ or $\mathrm{GaAs}$, having room temperature electron mobilities of 1450 and 8000 , respectively. We show how a high carrier density and high mobility can be achieved through the integration of an oxide heterostructure with a semiconductor.

In prior work, we demonstrated the integration of the structures LTO/STO and GTO/STO with Si, but the measured mobility is $<10 \mathrm{~cm}^{2} \mathrm{~V}^{-1} \mathrm{~s}^{-1}$ at room temperature [11-13]. In this work, we show using $\mathrm{x}$-ray photoemission (XPS) studies that a staggered conduction band alignment between the STO and $\mathrm{Si}$ creates a barrier to electrons, trapping the charge to the STO layer and resulting in low STO-like mobilities. Firstprinciples theory predicts that the addition of a monolayer of oxygen atoms at an STO-Si interface creates a dipole that moves the STO conduction band up by $\sim 0.5 \mathrm{eV}$ [14]. The consequence of this minimized conduction band offset (CBO) between STO and Si is a dramatic increase of the room temperature oxide mobility in oxide 2DEG heterostructures on $\mathrm{Si}$, improving from $\sim 1 \mathrm{~cm}^{2} \mathrm{~V}^{-1} \mathrm{~s}^{-1}$ in the as-grown samples

Published by the American Physical Society under the terms of the Creative Commons Attribution 4.0 International license. Further distribution of this work must maintain attribution to the author(s) and the published article's title, journal citation, and DOI. to over $100 \mathrm{~cm}^{2} \mathrm{~V}^{-1} \mathrm{~s}^{-1}$ in the oxygen treated samples, which we quantify by nonlinear Hall effect measurements.

The key idea is illustrated by examining the electrostatic consequences of band alignment using a 1D SchrödingerPoisson simulator (Fig. 1) for oxide 2DEGs integrated with silicon. Using the effective mass, dielectric constants, and band offsets of the constituent layers, an electron distribution and conduction band potential are calculated. Figure 1 shows the simulation for two heterostructures: one with a large CBO between the STO and Si and one with a smaller CBO. The integrated electron distribution in the Si layers is composed of $0.5 \%$ of the total charge distribution in the simulation with a CBO of $-0.7 \mathrm{eV}$ (here, negative CBO refers to the STO conduction band being below that of the $\mathrm{Si}$ ) and rises to $2.4 \%$ in the simulation with a smaller $\mathrm{CBO}$, an increase of $\sim 5$ times. An improved CBO between STO and Si reduces the electron confinement in the STO layer and results in charge transfer into the semiconductor channel. To change the band offset, we modify the oxygen content at the interface, which is predicted by theory to result in a $0.5 \mathrm{eV}$ increase of the STO-Si band offset.

\section{EXPERIMENTAL DETAILS}

Crystalline oxide integration on $\mathrm{Si}$ has been made possible with the development of STO/Si growth by molecular beam epitaxy (MBE) [15]. With a template STO layer, multifunctional oxide films and heterostructures that previously relied on ceramic bulk crystalline STO substrates can now be grown on an established Si platform, including piezoelectric $\mathrm{PbZrTiO}_{3}$ [16], ferroelectric $\mathrm{BaTiO}_{3}$ [17], ferromagnetic manganites [18], and cobaltates [19], multiferroic $\mathrm{BiFeO}_{3}$ [20], and high- $\kappa$ dielectrics such as $\mathrm{LaAlO}_{3}[21,22]$. STO/Si structures also demonstrate robustness for hydrogen production in photoelectrochemical water splitting $[23,24]$.

Oxide 2DEG heterostructures comprised of LTO/STO layers are grown by reactive oxide molecular beam epitaxy 

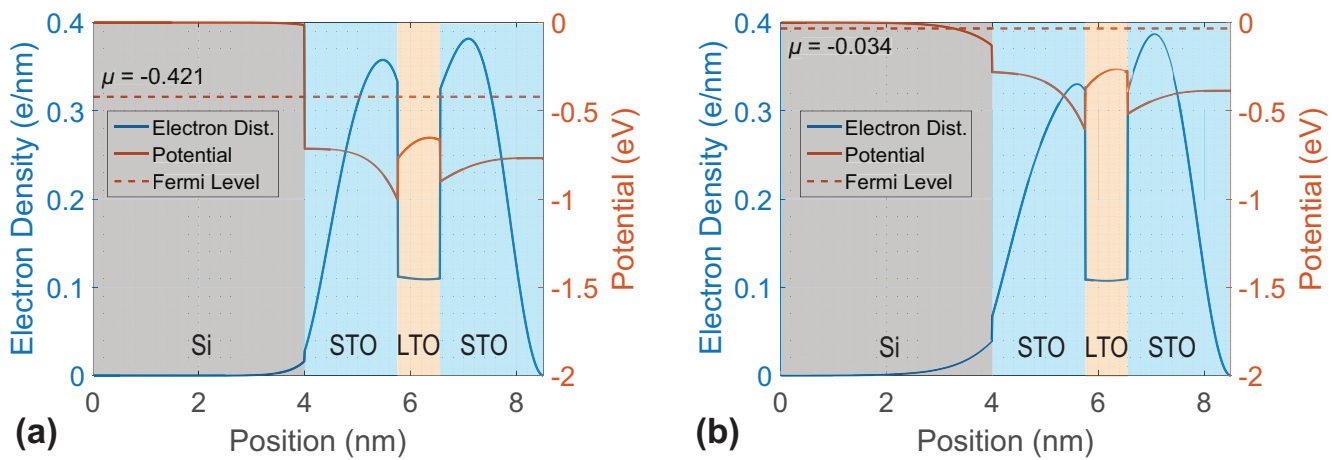

FIG. 1. Schrödinger-Poisson simulations of oxide 2DEG heterostructures on Si with different STO-Si band alignments. (a) Simulation showing a $-0.7 \mathrm{eV}$ CBO between STO and Si. (b) Simulation showing a smaller CBO of $-0.15 \mathrm{eV}$ between STO and Si. The structure is $1.95 \mathrm{~nm}(5 \mathrm{uc})$ of STO on $0.79 \mathrm{~nm}(2 \mathrm{uc})$ of LTO on $4 \mathrm{~nm}$ of Si. Band offsets of $0.25 \mathrm{eV}$ are used between the LTO and STO layers. The conduction band potential across the heterostructure is shown as a red curve, and the predicted electron distribution is shown as a blue curve.

(MBE) on insulating silicon substrates that are high purity, float zone 2-inch $\mathrm{Si}(001)$ wafers. Details of the growth are included in the Supplemental Material [25]. All structures in this study have a 4.5 unit cell (uc) STO template layer, which is the minimum required thickness to achieve continuous film coverage on Si. Carriers form in this STO template after epitaxial growth of a 2 uc LTO layer. These layers are capped by a 5 uc STO layer to prevent oxidation of the LTO. The oxygen stoichiometry at the STO/Si interface is modified by exposing the $4.5 \mathrm{uc}$ STO template layer to either molecular oxygen or to a radio frequency cavity generated oxygen plasma. Deposition of LTO and STO layers on top of the annealed 4.5 uc STO template layer follows the procedure reported in Ref. [11]. Band alignment measurements are carried out using x-ray photoemission spectroscopy measurements of STO/Si structures. Resistivity and Hall effect measurements are performed on squares cleaved from the Si wafers in the van der Pauw geometry.

\section{RESULTS AND DISCUSSION}

\section{A. Band alignment of oxygen-treated STO/Si}

Following the approach of Chambers et al. [26], the valence and conduction band alignments of a control sample, a $1800 \mathrm{~s}$ molecular oxygen annealed sample, a $30 \mathrm{~s}$ plasma annealed sample, and a $600 \mathrm{~s}$ plasma annealed sample are characterized. The band alignments of as-grown STO/Si samples and oxygen treated samples are summarized in Fig. 2. All anneals are performed at a substrate temperature of $\sim 550{ }^{\circ} \mathrm{C}$ as measured by pyrometry. The as-deposited sample with no additional oxygen treatment has a conduction band offset of $-0.7 \pm 0.15 \mathrm{eV}$, suggesting a barrier to the transfer of electrons from the LTO-doped STO to the Si. We note this is larger than what has been reported in other spectroscopy studies of STO/Si [26-28]. However, the introduction of oxygen through the different anneals all appear to raise the conduction band of the STO, as evidenced by a shift to lower binding energies for the Ti $3 p$ core level in the annealed samples. We measure a maximum increase of $0.56 \pm 0.05 \mathrm{eV}$ for the STO conduction band for the $30 \mathrm{~s}$ plasma annealed sample, in good agreement with the theoretical result of a $0.5 \mathrm{eV}$ increase.

Annealing in oxygen is expected to result in an increase in oxygen content at the interface to increase the CBO.
This increased oxygen content should be observed in XPS measurements of the $\mathrm{Si} 2 p$ peak, which exhibits a strong chemical shift as a function of $\mathrm{Si}$ oxidation state $[29,30]$. The amount of oxide at the $\mathrm{STO}-\mathrm{Si}$ interface, $\mathrm{SiO}_{x}$, is determined by fitting the area under the $\mathrm{SiO}_{x}$ subpeaks of the $\mathrm{Si} 2 p$ core level [Fig. 2(b)]. From the positon of the additional peaks, the oxidation state of the Si can be determined and is observed to increase, from between $\mathrm{Si}^{2+}$ and $\mathrm{Si}^{3+}$ in the molecular oxygen anneal, to between $\mathrm{Si}^{3+}$ and $\mathrm{Si}^{4+}$ for the plasma anneals. A maximum estimated $\mathrm{SiO}_{x}$ thickness of $\sim 4.3 \AA$ is observed for the most aggressive oxygen plasma anneal. Although the band offset does not change significantly between the different oxygen anneals, as seen from the lack of significant movement of the Ti $3 p$ peaks in Fig. 2(d), the difference spectra in Fig. 2(c) show that there is a marked difference in bonding at the STO-Si interface, with the plasma anneals showing higher $\mathrm{Si}$ oxidation states when compared to the molecular oxygen anneal. There may be an upper limit to the interfacial field formed from the introduction of oxygen, which would prevent further increase of the band offset despite a change in the chemical bonding. Longer anneals merely result in the growth of an unwanted amorphous $\mathrm{SiO}_{2}$ layer at the interface, which forms a barrier to electron transfer into the $\mathrm{Si}$. We now show through electrical transport measurements that the electrons in this channel reveal a higher electron mobility in the annealed oxide 2DEG structures on Si.

\section{B. Nonlinear Hall analysis of oxide 2DEGs on $\mathrm{Si}$}

To measure the consequences of the change in band offset on mobility, we use the Hall effect to measure carrier concentration and mobility. The analysis of the Hall data is complicated by the fact that the Hall resistance $R_{x y}(B)$ is observed to be nonlinear as a function of applied magnetic field for the oxygen annealed samples compared to the unannealed samples, indicating the presence of multiple conduction channels. For a single channel of transport, the Hall resistance has a linear dependence on magnetic field, $R_{x y}(B)=\frac{1}{n_{s} e}$, where $n_{s}$ is sheet carrier density (positive for holes and negative for electrons). The longitudinal resistance $R_{x x}$ has no dependence on $B$, and is related to the mobility $\mu$ by $R_{x x}=\frac{1}{n_{s} e \mu}$. On the other hand, a two-channel conduction model yields a nonlinear dependence on $B$ for both $R_{x y}$ and 
(a)

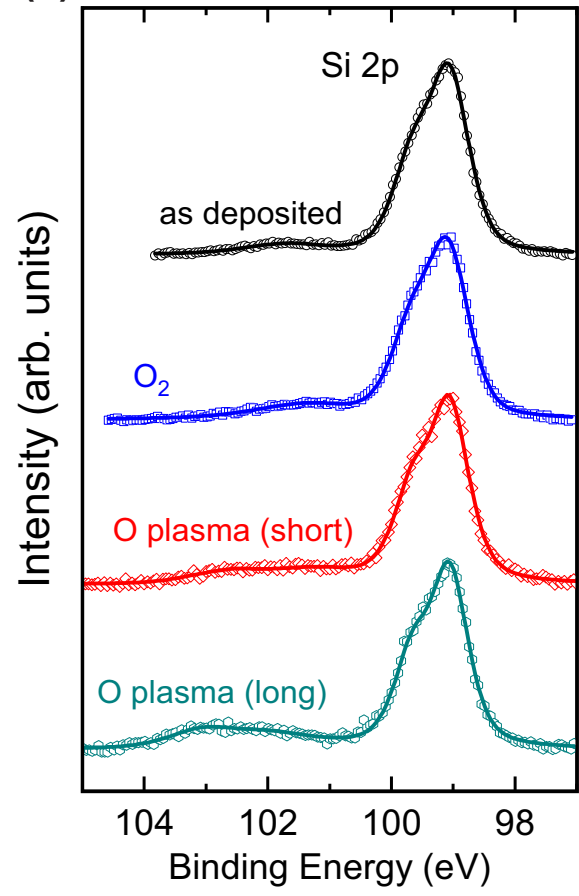

(b)

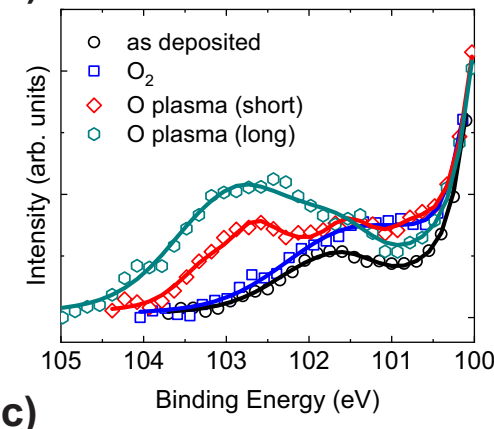

(c)

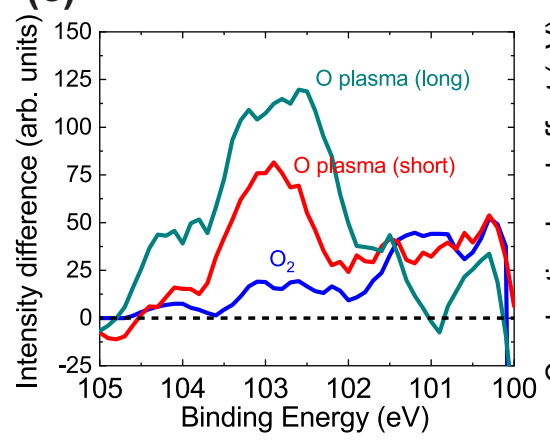

(d)

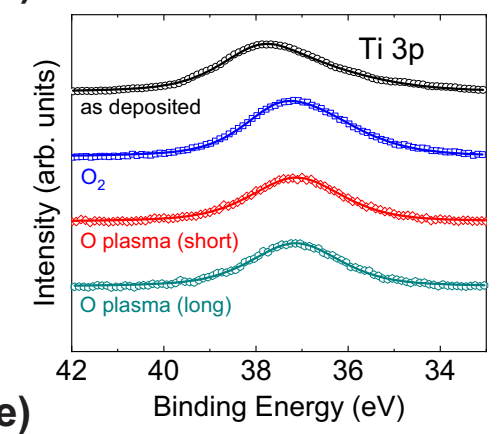

(e)

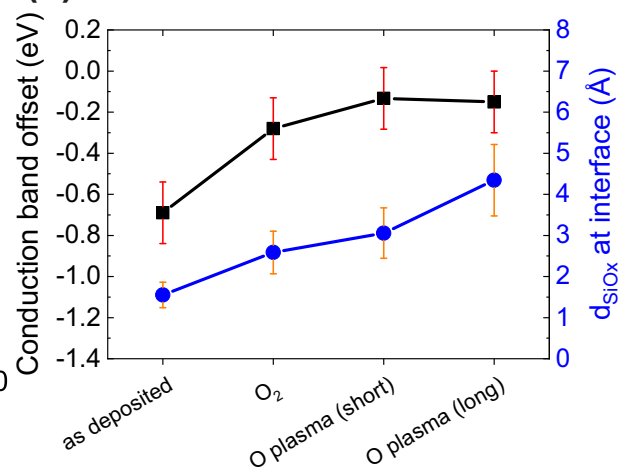

FIG. 2. XPS band alignment measurements of oxygen annealed STO/Si structures. (a) Spectra of Si $2 p$ core levels. The energies of the Si $2 p$ peaks are shifted to the as grown (control) sample. Raw data are shown as open symbols and lines are fits. "O ${ }_{2}$ " refers to samples annealed in molecular oxygen for $1800 \mathrm{~s}$, "short" refers to a 30 s plasma anneal, and "long" refers to a $600 \mathrm{~s}$ plasma anneal. (b) Expanded scale showing the shoulder near the $\mathrm{Si} 2 p$ peak, indicating the presence of interface $\mathrm{SiO}_{x}$ formation due to the oxygen anneals. (c) Intensity differences from (b) between the annealed samples and the unannealed sample (black dashed line). (d) Ti $3 p$ core level spectra. Gray arrows denote positions of peaks and show a shift to lower binding energy for the annealed samples. (e) Summary of calculated conduction band offsets between STO and $\mathrm{Si}$ (black squares) along with interface $\mathrm{SiO}_{x}$ thickness (blue circles) from XPS data. The values are plotted so that the anneals with the highest oxygen activity and time increase from left to right, and lines drawn in between data points serve as guides to the eye.

$R_{x x}$ [31]. Two-channel conduction models have been used to study transport in several thin film systems, including $\mathrm{MgB}_{2}$ [32] and oxide perovskites such as LTO/STO [33,34], LAO/STO [35-37], and $\mathrm{NdTiO}_{3} / \mathrm{STO}$ [38].

To account for the nonlinear Hall measurements for LTO/STO/Si heterostructures, we attribute one channel to the oxide layers near the STO/Si interface, which contain a high concentration of electrons $n_{o x}$ and a low mobility $\mu_{o x}$. A second channel lies in the Si bulk, with a lower electron density $n_{\mathrm{Si}}$ and a much higher mobility $\mu_{\mathrm{Si}}$. Two-channel fits to Hall data collected on these samples are shown in Figs. 3 and 4 (see Supplemental Material [25] for details). The unannealed samples show linear Hall behavior, with an electron density of $5-6 \times 10^{14} \mathrm{~cm}^{-2}$, an electron mobility of $\sim 1 \mathrm{~cm}^{2} \mathrm{~V}^{-1} \mathrm{~s}^{-1}$, and little temperature dependence. The annealed samples are nonlinear in $R_{x y}(B)$ at most temperatures, with the $\mathrm{Si}$ channel having a low density of $\sim 10^{10}-10^{11} \mathrm{~cm}^{-2}$ at room temperature that exponentially decreases as the temperature is lowered, characteristic of unintentionally doped carriers in the Si bulk.

For the unannealed films, the measured carrier concentration and mobility are characteristic of the formation of a 2DEG due to the polar discontinuity at the two LTO/STO interfaces [1,2], with a characteristically low mobility of $1 \mathrm{~cm}^{2} \mathrm{~V}^{-1} \mathrm{~s}^{-1}$. Surprisingly, the measured mobility jumps for the annealed films by up to two orders of magnitude. We believe that the spatial distribution of $n_{o x}$ shifts from the STO layers in the unannealed sample to a region in the interface between the STO and Si layers for the annealed samples. This increase in mobility is a consequence of the increase in $\mathrm{CBO}$ and carriers moving into the silicon from the LTO/STO 2DEG, as shown in Fig. 1. For an STO/Si CBO of $-0.15 \mathrm{eV}$ (the highest measured value in our anneals), the SchrödingerPoisson simulations predict that the Si layers have a $2 \mathrm{DEG}$ density of $1.6 \times 10^{13} \mathrm{~cm}^{-2}$, which is found in the tail of the electron distribution that penetrates deeper into the $\mathrm{Si}$ layers. Because a high density of carriers is found in the $\mathrm{Si}$, the measured mobility is high, up to $110 \pm 30 \mathrm{~cm}^{2} \mathrm{~V}^{-1} \mathrm{~s}^{-1}$, having a value similar to that expected from an extrapolation of the universal mobility curve for $\mathrm{Si}$ to a field $(1.7 \mathrm{MV} / \mathrm{cm})$ that is consistent with the measured carrier concentration of $1.1 \pm 0.25 \times 10^{13} \mathrm{~cm}^{-2}[39]$.

We note that our picture of the electronic structure shown in Fig. 1(b) includes a third channel of charge in the low mobility oxide 2DEG (of order $\sim 10^{14} \mathrm{~cm}^{-2}$ ). However, because the mobility of this layer is expected to be small relative to the maximum magnetic field applied, the Hall resistance cannot resolve the two low mobility contributions. We believe a twocarrier Hall model is as far as we can meaningfully analyze the data to capture the electrical transport properties. Qualitatively, we observe a temperature dependence to the mobility for the higher oxide mobility samples from room temperature 

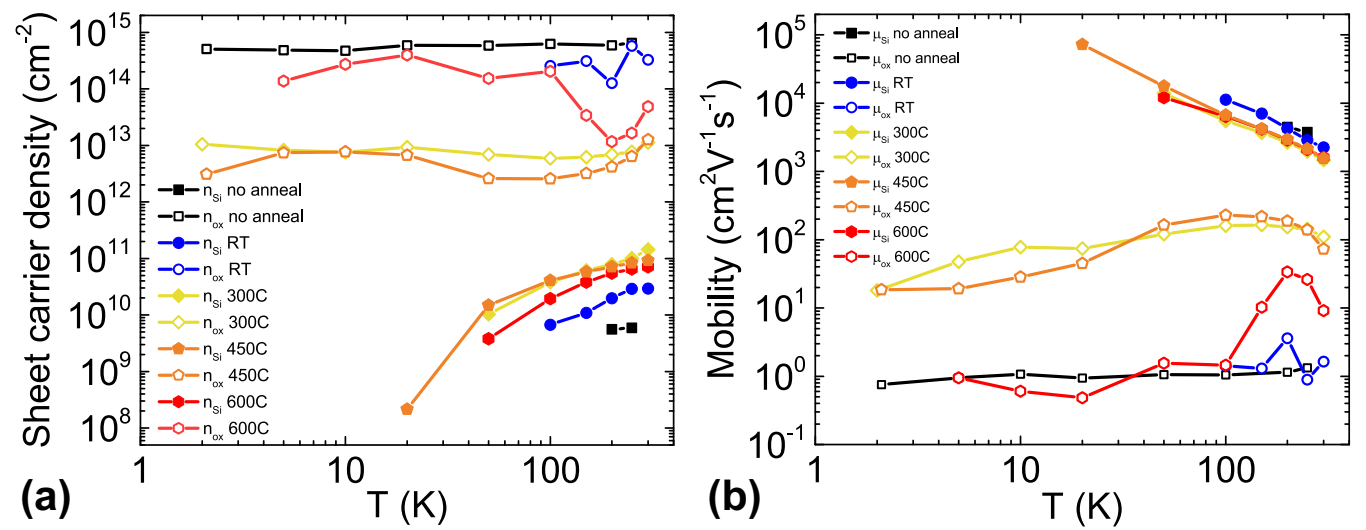

FIG. 3. Two-channel Hall fits to $n_{o x}, n_{\mathrm{Si}}, \mu_{o x}$, and $\mu_{\mathrm{Si}}$ for oxygen plasma annealed STO/LTO/STO/Si heterostructures. (a) Sheet carrier density as a function of measurement temperature. (b) Electron mobility as a function of measurement temperature. Filled shapes represent transport in the bulk Si channel, and open shapes represent transport through the oxide layer close to the STO/Si interface. Anneals are performed on the $4.5 \mathrm{uc} \mathrm{STO} / \mathrm{Si}$ template layer for 10 minutes and range from room temperature (RT) to $600{ }^{\circ} \mathrm{C}$. Unannealed control samples are also shown (black squares). Lines are meant as guides to the eye. The unannealed sample shows a linear Hall signal $<200 \mathrm{~K}$. The annealed samples show nonlinear Hall signals from room temperature down to $<100 \mathrm{~K}$. In the linear regime, transport is associated with a single channel in the oxide layer. Linear fits have errors smaller than $3 \%$, whereas nonlinear fits have errors ranging from 15 to $30 \%$.

down to $\sim 100 \mathrm{~K}$ that is consistent with signatures of phonon scattering in both STO [10,40] as well as in highly doped $\mathrm{Si}$. We also observe that the mobility peaks at $\sim 100-200 \mathrm{~K}$ and falls off at lower temperatures, which may be evidence of charged impurity scattering observed in semiconductors [41], perhaps coming from the interface or STO.

To determine whether the mobility in the oxide channel can be further optimized, a series of oxygen anneals of varying duration is performed at a substrate temperature of $300{ }^{\circ} \mathrm{C}$, and transport data from Hall measurements are summarized in Fig. 4. There is a trend of increasing mobility in the oxide channel as the anneal duration increases, while the carrier density in the oxide channel correspondingly decreases. This is consistent with the photoemission data shown in Fig. 2, which shows that the band offset between $\mathrm{STO}$ and $\mathrm{Si}$ is reduced as more oxygen is introduced into the structure. However, at the 10-minute mark for anneal duration, spectroscopy reveals that the introduced oxygen species starts back bonding to the $\mathrm{Si}$ lattice to form $\mathrm{SiO}_{2}$ at the interface. Longer anneals will hinder device performance due to the creation of an insulating barrier at the STO/Si interface for carrier transport. Longer anneal duration can also hinder transport due to degradation of the oxide-semiconductor interface quality. We conclude that an optimal anneal of 10 minutes at a substrate temperature of $300^{\circ} \mathrm{C}$ improves the mobility of the oxide 2DEG heterostructure on Si by two orders of magnitude, from $\sim 1 \mathrm{~cm}^{2} \mathrm{~V}^{-1} \mathrm{~s}^{-1}$ to $>100 \mathrm{~cm}^{2} \mathrm{~V}^{-1} \mathrm{~s}^{-1}$, while avoiding substantial interfacial $\mathrm{SiO}_{2}$ formation.

\section{SUMMARY}

We have demonstrated an approach to increase the room temperature oxide mobility in STO-based 2DEG heterostructures integrated on $\mathrm{Si}$ in order to realize high
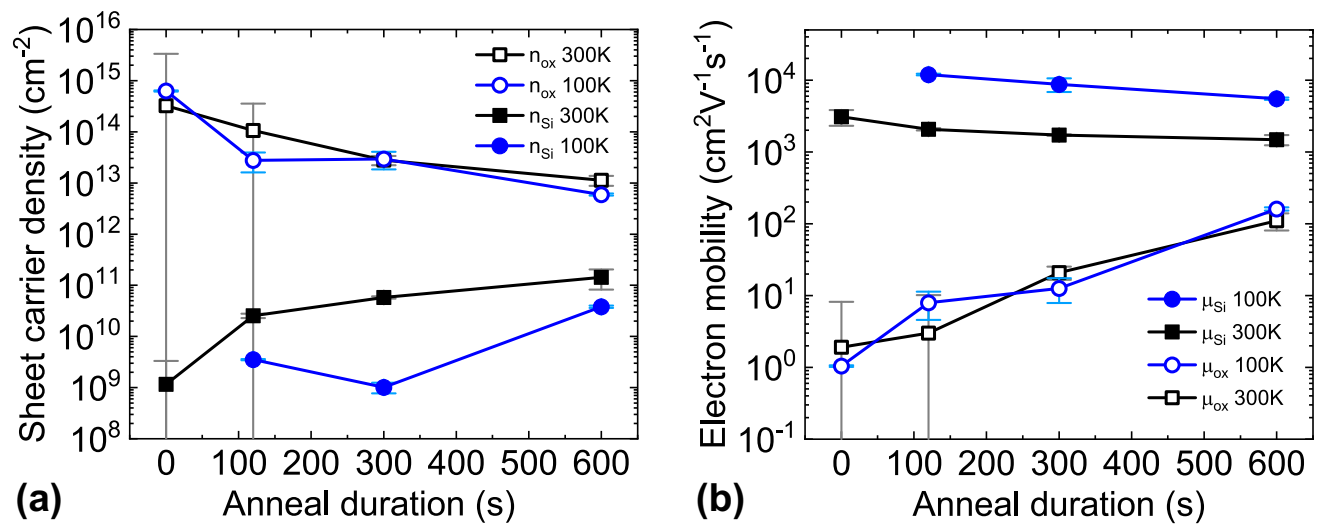

FIG. 4. Study of oxide and silicon channel (a) carrier densities and (b) carrier mobilities as a function of oxygen plasma anneal length. The heterostructures consist of 5 uc STO/2 uc LTO/4.5 uc STO deposited on undoped Si. The template 4.5 uc STO/Si is annealed at $300{ }^{\circ} \mathrm{C}$ in oxygen plasma for different durations ranging from $0 \mathrm{~s}$ (i.e., unannealed) to $600 \mathrm{~s}$. Two-channel Hall fits to $n_{o x}, n_{\mathrm{Si}}, \mu_{o x}$, and $\mu_{\mathrm{Si}}$ are plotted, with $\mathrm{Si}$ channel data shown as filled shapes and oxide channel data shown as open shapes. Measurement temperatures of room temperature $(300 \mathrm{~K})$ and low temperature $(100 \mathrm{~K})$ are taken. Thick lines connecting data points are shown as guides to the eye. Error bars are shown in gray for the fits to the $300 \mathrm{~K}$ data and in light blue for fits to the low temperature data. 
carrier concentration and high mobility structures for applications based on a scalable semiconductor platform. XPS measurements of STO/Si conduction band alignments reveal that the conduction band of STO is $0.7 \mathrm{eV}$ below that of the $\mathrm{Si}$, which results in carrier confinement in the oxide layers. Guided by first principle calculations, we show that the band offset can be reduced by $0.56 \mathrm{eV}$ via the introduction of a thin oxide layer at the $\mathrm{STO} / \mathrm{Si}$ interface, which is predicted to create an interface dipole that lifts the STO conduction band. We confirm this prediction with XPS measurements on oxygen-annealed structures. Schrödinger-Poisson simulations show that the reduction of the STO/Si CBO leads to an increase of charge transferred into the Si layers, which results in higher electron mobilities. Hall measurements quantify two channels of conduction and show a carrier density of $1.1 \times 10^{13} \mathrm{~cm}^{-2}$ with an electron mobility of $110 \mathrm{~cm}^{2} \mathrm{~V}^{-1} \mathrm{~s}^{-1}$ in the annealed structures. This represents a substantial im- provement in mobility for oxide electronics, and is an order of magnitude higher than what has been measured in oxide-only materials. This approach of remotely doping semiconductors from oxide 2DEGs serves as an approach toward the control of electronic transport in oxide electronics.

\section{ACKNOWLEDGMENTS}

This work was funded by the Office of Naval Research Multidisciplinary University Research Initiative to support the EXtreme Electron DEvices (EXEDE) program. A.K. and S.I.B. acknowledge partial support by the NSF via Grant No. MRSEC DMR 1119826. Facility support was provided by the NSF under Grant No. MRSEC DMR 1119826. We thank Susanne Stemmer, Siddharth Rajan, and Lior Kornblum for valuable discussions.
[1] A. Ohtomo and H. Y. Hwang, Nature (London) 427, 423 (2004).

[2] P. Moetakef, T. A. Cain, D. G. Ouellette, J. Y. Zhang, D. O. Klenov, A. Janotti, C. G. Van De Walle, S. Rajan, S. J. Allen, and S. Stemmer, Appl. Phys. Lett. 99, 232116 (2011).

[3] P. Xu, D. Phelan, J. Seok Jeong, K. Andre Mkhoyan, and B. Jalan, Appl. Phys. Lett. 104, 082109 (2014).

[4] K. Shibuya, T. Ohnishi, M. Kawasaki, H. Koinuma, and M. Lippmaa, Jpn. J. Appl. Phys. 43, L1178 (2004).

[5] J. Y. Zhang, C. A. Jackson, R. Chen, S. Raghavan, P. Moetakef, L. Balents, and S. Stemmer, Phys. Rev. B 89, 075140 (2014).

[6] P. Xu, Y. Ayino, C. Cheng, V. S. Pribiag, R. B. Comes, P. V. Sushko, S. A. Chambers, and B. Jalan, Phys. Rev. Lett. 117, 106803 (2016).

[7] M. Boucherit, O. Shoron, C. A. Jackson, T. A. Cain, M. L. C. Buffon, C. Polchinski, S. Stemmer, and S. Rajan, Appl. Phys. Lett. 104, 182904 (2014).

[8] G. Herranz, M. Basletić, M. Bibes, C. Carrétéro, E. Tafra, E. Jacquet, K. Bouzehouane, C. Deranlot, A. Hamzić, J.-M. Broto, A. Barthélémy, and A. Fert, Phys. Rev. Lett. 98, 216803 (2007).

[9] J. Son, P. Moetakef, B. Jalan, O. Bierwagen, N. J. Wright, R. Engel-Herbert, and S. Stemmer, Nat. Mater. 9, 482 (2010).

[10] A. Verma, A. P. Kajdos, T. A. Cain, S. Stemmer, and D. Jena, Phys. Rev. Lett. 112, 216601 (2014).

[11] E. N. Jin, L. Kornblum, D. P. Kumah, K. Zou, C. C. Broadbridge, J. H. Ngai, C. H. Ahn, and F. J. Walker, APL Mater. 2, 116109 (2014).

[12] L. Kornblum, E. N. Jin, D. P. Kumah, A. T. Ernst, C. C. Broadbridge, C. H. Ahn, and F. J. Walker, Appl. Phys. Lett. 106, 201602 (2015).

[13] E. N. Jin, L. Kornblum, C. H. Ahn, and F. J. Walker, MRS Adv. 1, 287 (2016).

[14] A. M. Kolpak and S. Ismail-Beigi, Phys. Rev. B 85, 195318 (2012).

[15] R. A. McKee, F. J. Walker, and M. F. Chisholm, Phys. Rev. Lett. 81, 3014 (1998).

[16] A. Lin, X. Hong, V. Wood, A. A. Verevkin, C. H. Ahn, R. A. McKee, F. J. Walker, and E. D. Specht, Appl. Phys. Lett. 78, 2034 (2001).

[17] V. Vaithyanathan, J. Lettieri, W. Tian, A. Sharan, A. Vasudevarao, Y. L. Li, A. Kochhar, H. Ma, J. Levy, P. Zschack, J. C.
Woicik, L. Q. Chen, V. Gopalan, and D. G. Schlom, J. Appl. Phys. 100, 024108 (2006).

[18] A. K. Pradhan, J. B. Dadson, D. Hunter, K. Zhang, S. Mohanty, E. M. Jackson, B. Lasley-Hunter, K. Lord, T. M. Williams, R. R. Rakhimov, J. Zhang, D. J. Sellmyer, K. Inaba, T. Hasegawa, S. Mathews, B. Joseph, B. R. Sekhar, U. N. Roy, Y. Cui, and A. Burger, J. Appl. Phys. 100, 033903 (2006).

[19] A. Posadas, M. Berg, H. Seo, A. de Lozanne, A. A. Demkov, D. J. Smith, A. P. Kirk, D. Zhernokletov, and R. M. Wallace, Appl. Phys. Lett. 98, 053104 (2011).

[20] J. Wang, H. Zheng, Z. Ma, S. Prasertchoung, M. Wuttig, R. Droopad, J. Yu, K. Eisenbeiser, and R. Ramesh, Appl. Phys. Lett. 85, 2574 (2004).

[21] J. W. Reiner, A. Posadas, M. Wang, T. P. Ma, and C. H. Ahn, Microelectron. Eng. 85, 36 (2008).

[22] Y. Y. Mi, Z. Yu, S. J. Wang, P. C. Lim, Y. L. Foo, A. C. H. Huan, and C. K. Ong, Appl. Phys. Lett. 90, 181925 (2007).

[23] L. Ji, M. D. McDaniel, S. Wang, A. B. Posadas, X. Li, H. Huang, J. C. Lee, A. A. Demkov, A. J. Bard, J. G. Ekerdt, and E. T. Yu, Nat. Nanotechnol. 10, 84 (2015).

[24] H. Seo, A. B. Posadas, C. Mitra, A. V. Kvit, J. Ramdani, and A. A. Demkov, Phys. Rev. B 86, 075301 (2012).

[25] See Supplemental Material at http://link.aps.org/supplemental/ 10.1103/PhysRevMaterials.2.115001 for epitaxial growth details, sheet resistance data, a description of the nonlinear Hall effect data fits, and a comparison between a two-carrier and three-carrier transport model.

[26] S. A. Chambers, Y. Liang, Z. Yu, R. Droopad, J. Ramdani, and K. Eisenbeiser, Appl. Phys. Lett. 77, 1662 (2000).

[27] F. Amy, A. S. Wan, A. Kahn, F. J. Walker, and R. A. McKee, J. Appl. Phys. 96, 1635 (2004).

[28] S. A. Chambers, Y. Liang, Z. Yu, R. Droopad, and J. Ramdani, J. Vac. Sci. Technol. A 19, 934 (2001).

[29] F. J. Himpsel, F. R. McFeely, A. Taleb-Ibrahimi, J. A. Yarmoff, and G. Hollinger, Phys. Rev. B 38, 6084 (1988).

[30] Z. H. Lu, M. J. Graham, D. T. Jiang, and K. H. Tan, Appl. Phys. Lett. 63, 2941 (1993).

[31] N. W. Ashcroft and N. D. Mermin, Solid State Physics (Brooks/Cole, Belmont, CA, 1976). 
[32] H. Yang, Y. Liu, C. Zhuang, J. Shi, Y. Yao, S. Massidda, M. Monni, Y. Jia, X. Xi, Q. Li, Z.-K. Liu, Q. Feng, and H.-H. Wen, Phys. Rev. Lett. 101, 067001 (2008).

[33] J. S. Kim, S. S. A. Seo, M. F. Chisholm, R. K. Kremer, H.-U. Habermeier, B. Keimer, and H. N. Lee, Phys. Rev. B 82, 201407 (2010).

[34] R. Ohtsuka, M. Matvejeff, K. Nishio, R. Takahashi, and M. Lippmaa, Appl. Phys. Lett. 96, 192111 (2010).

[35] V. K. Guduru, A. McCollam, J. C. Maan, U. Zeitler, S. Wenderich, M. K. Kruize, A. Brinkman, M. Huijben, G. Koster, D. H. A. Blank, G. Rijnders, and H. Hilgenkamp, J. Korean Phys. Soc. 63, 437 (2013).

[36] R. Pentcheva, M. Huijben, K. Otte, W. E. Pickett, J. E. Kleibeuker, J. Huijben, H. Boschker, D. Kockmann, W.
Siemons, G. Koster, H. J. W. Zandvliet, G. Rijnders, D. H. A. Blank, H. Hilgenkamp, and A. Brinkman, Phys. Rev. Lett. 104, 166804 (2010).

[37] Z. Chen, H. Yuan, Y. Xie, D. Lu, H. Inoue, Y. Hikita, C. Bell, and H. Y. Hwang, Nano Lett. 16, 6130 (2016).

[38] P. Xu, T. C. Droubay, J. S. Jeong, K. A. Mkhoyan, P. V. Sushko, S. A. Chambers, and B. Jalan, Adv. Mater. Interfaces 3, 1500432 (2016).

[39] N. Arora, MOSFET Models for VLSI Circuit Simulation (Springer-Verlag, Vienna, 1993).

[40] E. Mikheev, C. R. Freeze, B. J. Isaac, T. A. Cain, and S. Stemmer, Phys. Rev. B 91, 165125 (2015).

[41] D. Long and J. Myers, Phys. Rev. 115, 1107 (1959). 